The Astrophysical Journal, 629:L89-L92, 2005 August 20

(C) 2005. The American Astronomical Society. All rights reserved. Printed in U.S.A.

\title{
HUBBLE SPACE TELESCOPE IMAGING OF THE ULTRACOMPACT BLUE DWARF GALAXY HS 0822+3542: AN ASSEMBLING GALAXY IN A LOCAL VOID?
}

\author{
Michael R. Corbin, ${ }^{1}$ William D. Vacca, ${ }^{2}$ John E. Hibbard, ${ }^{3}$ Rachel S. Somerville, ${ }^{4}$ And Rogier A. Windhorst ${ }^{1}$ \\ Received 2005 May 20; accepted 2005 July 22; published 2005 August 4
}

\begin{abstract}
We present deep $U$-band, narrow $V$-band, and $I$-band images and photometry of the ultracompact blue dwarf galaxy HS 0822+3542 obtained with the Advanced Camera for Surveys/High Resolution Channel of the Hubble Space Telescope. This object is also extremely metal-poor $[12+\log (\mathrm{O} / \mathrm{H})=7.45]$ and resides in a nearby void. The images resolve it into two physically separate components that were previously described as star clusters in a single galaxy. The primary component is only $\sim 100 \mathrm{pc}$ in maximum extent and consists of a starburst region surrounded by a ringlike structure of relatively redder stars. The secondary component is $\sim 50$ pc in size and lies at a projected distance of $\sim 80 \mathrm{pc}$ away from the primary, and is also actively star-forming. We estimate masses of $\sim 10^{7}$ and $\sim 10^{6} M_{\odot}$ for the two components based on their luminosities, with an associated dynamical timescale for the system of a few megayears. This timescale and the structure of the components suggest that a collision between them triggered their starbursts. The spectral energy distributions of both components can be fitted by the combination of a recent (a few megayears old) starburst and an evolved (several gigayears old) underlying stellar population, similar to larger blue compact dwarf galaxies. This indicates that despite its metal deficiency, the object is not forming its first generation of stars. However, the small sizes and masses of the two components suggest that HS $0822+3542$ represents a dwarf galaxy in the process of assembling from clumps of stars intermediate in size between globular clusters and objects previously classified as galaxies. Its relatively high ratio of neutral gas mass to stellar mass $(\sim 1)$ and high specific star formation rate, $\log \left(\mathrm{SFR} / M_{\odot}\right) \simeq-9.2$, also suggest that it is still converting much of its gas to stars.
\end{abstract}

Subject headings: galaxies: dwarf — galaxies: formation — galaxies: individual (HS 0822+3542) galaxies: starburst — galaxies: stellar content

\section{INTRODUCTION}

The blue compact dwarf galaxy HS $0822+3542$ (=SDSS $\mathrm{J} 082555.44+353231.9)$ is one of the most metal-poor galaxies known, having $12+\log (\mathrm{O} / \mathrm{H})=7.45 \pm 0.02$ as measured from its strong nebular emission lines (Kniazev et al. 2000, 2003). Its redshift of $z=0.00233$ (Kniazev et al. 2003) corresponds to a distance of only $12.7 \mathrm{Mpc}$ under a cosmology of $H_{0}=71 \mathrm{~km} \mathrm{~s}^{-1} \mathrm{Mpc}^{-1}, \Omega_{\Lambda}=0.73$, and $\Omega_{M}=0.27$, after correction to the frame of the cosmic microwave background. It is, however, very faint and blue, with a Sloan Digital Sky Survey $^{5} g$ magnitude of $17.64\left(M_{g}=-12.88\right)$ and $g-r=$ -0.08 . HS $0822+3542$ is also "ultracompact" under the criteria of having an angular size of just a few arcseconds, as measured from ground-based images (Kniazev et al. 2000; Gil de Paz et al. 2003), and a physical size of less than $1 \mathrm{kpc}$. It resides in a void in the direction of Lynx-Cancer, with the nearest large galaxy more than $3 \mathrm{Mpc}$ away (Pustilnik et al. 2003, hereafter P03). P03 suggest that its star formation has been triggered by interaction with the low surface brightness dwarf galaxy SAO $0822+3545$, located at a projected distance of 3.5 $(13 \mathrm{kpc})$ away and having a redshift difference of only $\sim 22 \mathrm{~km} \mathrm{~s}^{-1}$.

In this Letter we present images and photometry of HS $0822+3542$ obtained with the Hubble Space Telescope (HST) Advanced Camera for Surveys/High Resolution Channel (ACS/

\footnotetext{
${ }^{1}$ Department of Physics and Astronomy, Arizona State University, P.O. Box 871504, Tempe, AZ 85287; michael.corbin@asu.edu, rogier.windhorst@asu.edu.

${ }^{2}$ Universities Space Research Association, MS 144-2, NASA/Ames Research Center, Mountain View, CA 94025; wvacca@mail.arc.nasa.gov.

${ }^{3}$ National Radio Astronomy Observatory, 520 Edgemont Road., Charlottesville, VA 22903; jhibbard@nrao.edu.

${ }^{4}$ Space Telescope Science Institute, 3700 San Martin Drive, Baltimore, MD 21218; somerville@stsci.edu.

${ }^{5}$ See http://www.sdss.org.
}

HRC). Our data on HS $0822+3542$ are part of a larger program to obtain ACS/HRC images of a sample of ultracompact blue dwarf galaxies (UCBDs) selected from the Sloan Digital Sky Survey. Our primary goal is to resolve the structure of these objects in order to test the hypothesis that they are very small dwarf galaxies in the process of assembling from smaller clumps of gas and stars, as appears to be the case for the prototypical UCBD, POX 186 (Corbin \& Vacca 2002). Our results for HS $0822+3542$ support this hypothesis, as the ACS/ HRC images resolve the galaxy into two physically separate but very small $(\sim 100 \mathrm{pc})$ clumps of stars with complex internal structure that may ultimately coalesce and form a single quiescent dwarf galaxy. These clumps were also detected in the deconvolved ground-based images of P03 but were interpreted as star clusters within a single galaxy. We also find evidence of an underlying old ( $10 \mathrm{Gyr})$ stellar population within both clumps of stars, indicating that despite its metal deficiency, the object is not forming its first generation of stars.

\section{OBSERVATIONS AND RESULTS}

\subsection{HST Observations}

HST ACS/HRC observations of HS $0822+3542$ were made on UT 2004 October 4. The filters and total integration times were F330W ( $U ; 1666$ s), F550M ("narrow $V$ "; 1906 s), and $\mathrm{F} 814 \mathrm{~W}(I ; 1366 \mathrm{~s})$. The limiting $3 \sigma \mathrm{AB}$ magnitudes for each final image are approximately 25.9 (F330W), 25.7 (F550M), and $26.5(\mathrm{~F} 814 \mathrm{~W})$. These filters were chosen to cover a wide wavelength range and to exclude strong emission lines such as [O II] $\lambda 3727$, [O III] $\lambda \lambda 4959,5007$, and $\mathrm{H} \alpha$. The F814W filter contains the lines He I $\lambda 7065$ and [Ar III] $\lambda 7136$, but from the measured fluxes of these lines in P03 we estimate their contribution to the total flux measured in the filter to be $<1 \%$. The 


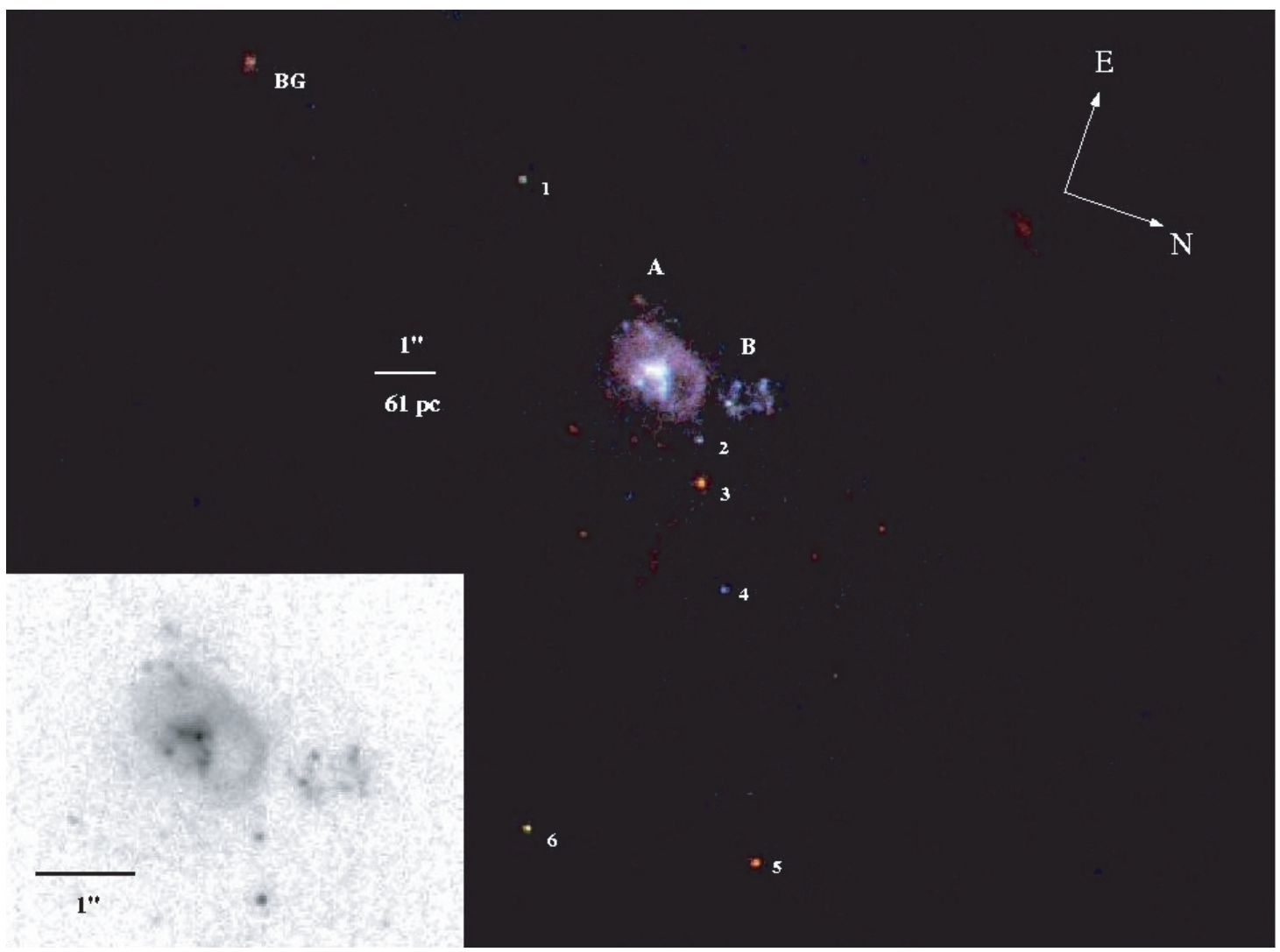

FIG. 1.-Color image of HS $0822+3542$ created from the ACS/HRC F330W, F550M, and F814W images and displayed on a logarithmic intensity scale. The object labeled "BG" is a probable background galaxy. The objects labeled 1-6 are point sources. The inset shows a gray-scale version of the color image in the region of components $\mathrm{A}$ and $\mathrm{B}$ to better show their internal structure.

images were taken in a two-point dither pattern to optimize cosmic-ray rejection, and the subimages in each filter were combined with the STSDAS task MULTIDRIZZLE to produce final cleaned images at the nominal ACS/HRC resolution of 0 ".027 pixel $^{-1}$. We measure the FWHM of the point sources in the images to be approximately 2.8 pixels, corresponding to a resolution of approximately $5 \mathrm{pc}$ at the adopted distance to the galaxy. A correction for Galactic foreground extinction was applied to each image based on the maps of Schlegel et al. (1998). From the Sloan Digital Sky Survey spectrum of the galaxy (see Kniazev et al. 2003), we measure a very small amount of internal extinction, $E(B-V) \simeq 0.04$, indicating little internal dust. We also confirm the Kniazev et al. (2003) metallicity value of $12+\log (\mathrm{O} / \mathrm{H})=7.45 \pm 0.02$ to within uncertainties.

Figure 1 presents a color composite of the F330W, F550M, and F814W images. It also shows a gray-scale version of this image centered on the central part of the object in order to better display its internal structure. We confirm the result of P03 that the brightest portion of the object consists of two components, which we designate as $\mathrm{A}$ and $\mathrm{B}$ (corresponding to their components "a" and "b"). We resolve their component "c" into a single star, and their component "d" into two separate stars whose absolute magnitudes (see $\S 2.2$ ) indicate them to be supergiant stars associated with the galaxy. Our main finding, however, is that $\mathrm{A}$ and $\mathrm{B}$ are not individual star clusters within a larger galaxy, as discussed by P03, but are physically separate objects with complex internal structure. Component A consists of a central starburst region surrounded by a ringlike structure of relatively redder stars, while component B is more irregular. The angular sizes of both components are also very small, corresponding to small physical sizes at the adopted distance to the galaxy of $12.7 \mathrm{Mpc}$. The major axis of component A is only $\sim 1$ ".62 (100 pc), while that of component B is only $\sim 0$ ".78 $(50 \mathrm{pc})$. The faint plume of stars to the northwest of A and B seen in the ground-based images of HS $0822+3542$ (see Kniazev et al. 2000, P03, and Gil de Paz et al. 2003) is detected in all of the images after smoothing to a resolution comparable to the ground-based images of the object, $\sim 0$ ".6 pixel $^{-1}$. The maximum extent of the object including the faint plume is $\sim 400 \mathrm{pc}$, making it comparable in size to POX 186 (Corbin \& Vacca 2002).

\subsection{Photometry and Comparison with Population Synthesis Models}

We performed photometry on components A and B using the IRAF task POLYPHOT, fitting polygonal apertures around them, setting a sky annulus approximately 6" away from their centers, and converting from count rates to fluxes using the conversion factors in the image headers. We additionally performed photometry on the brighter point sources in the images, numbered on Figure 1. The resulting AB magnitudes and colors are presented in Table 1 , along with the associated absolute magnitudes in the F550M band. The absolute magnitudes of the point sources all fall in the range of supergiant stars under the assumption that they lie at the adopted distance to the galaxy of $12.7 \mathrm{Mpc}$. The flux in the starburst region at the center of 
TABLE 1

AB Magnitudes and Colors

\begin{tabular}{|c|c|c|c|c|}
\hline Object & F550M & F330W - F550M & $\mathrm{F} 550 \mathrm{M}-\mathrm{F} 814 \mathrm{~W}$ & $M_{\mathrm{F} 550 \mathrm{M}}{ }^{\mathrm{a}}$ \\
\hline A & $19.55 \pm 0.08$ & $-0.46 \pm 0.16$ & $-0.07 \pm 0.02$ & $-11.02 \pm 0.08$ \\
\hline B $\ldots \ldots$ & $21.37 \pm 0.18$ & $-0.47 \pm 0.27$ & $-0.07 \pm 0.04$ & $-9.15 \pm 0.18$ \\
\hline 1 & $24.3 \pm 0.7$ & $1.2 \pm 2.2$ & $-0.4 \pm 1.3$ & $-6.2 \pm 0.7$ \\
\hline 2 & \pm 0.7 & \pm 1.5 & \pm 1.2 & \pm 0.7 \\
\hline 3 & \pm 0.7 & \pm 0.7 & \pm 1.0 & \pm 0.7 \\
\hline 4 & \pm 1.0 & \pm 1.9 & \pm 1.5 & \pm 1.0 \\
\hline $5 \ldots \ldots$ & \pm 0.9 & \pm 0.9 & \pm 1.2 & \pm 0.9 \\
\hline $6 \ldots \ldots$ & \pm 0.7 & \pm 0.7 & \pm 1.2 & \pm 0.7 \\
\hline
\end{tabular}

${ }^{a}$ Calculated for a distance of $12.7 \mathrm{Mpc}(\S 1)$.

component A corresponds to an equivalent of $\sim 55$ OB stars, which appear to be collected into a central bright cluster with approximately five to six smaller associations nearby (Fig. 1). The central cluster thus falls short of being a "super" star cluster under the criterion of having a mass $\sim 10^{4} M_{\odot}$ (see Whitmore 2003 and references therein). The brighter point sources in region B may represent individual stars or small OB clusters or associations.

Figure 2 shows the fluxes of components $\mathrm{A}$ and $\mathrm{B}$ as measured in all three filters. The combined F550M filter flux of components A and B agrees with the flux level of the spectrum
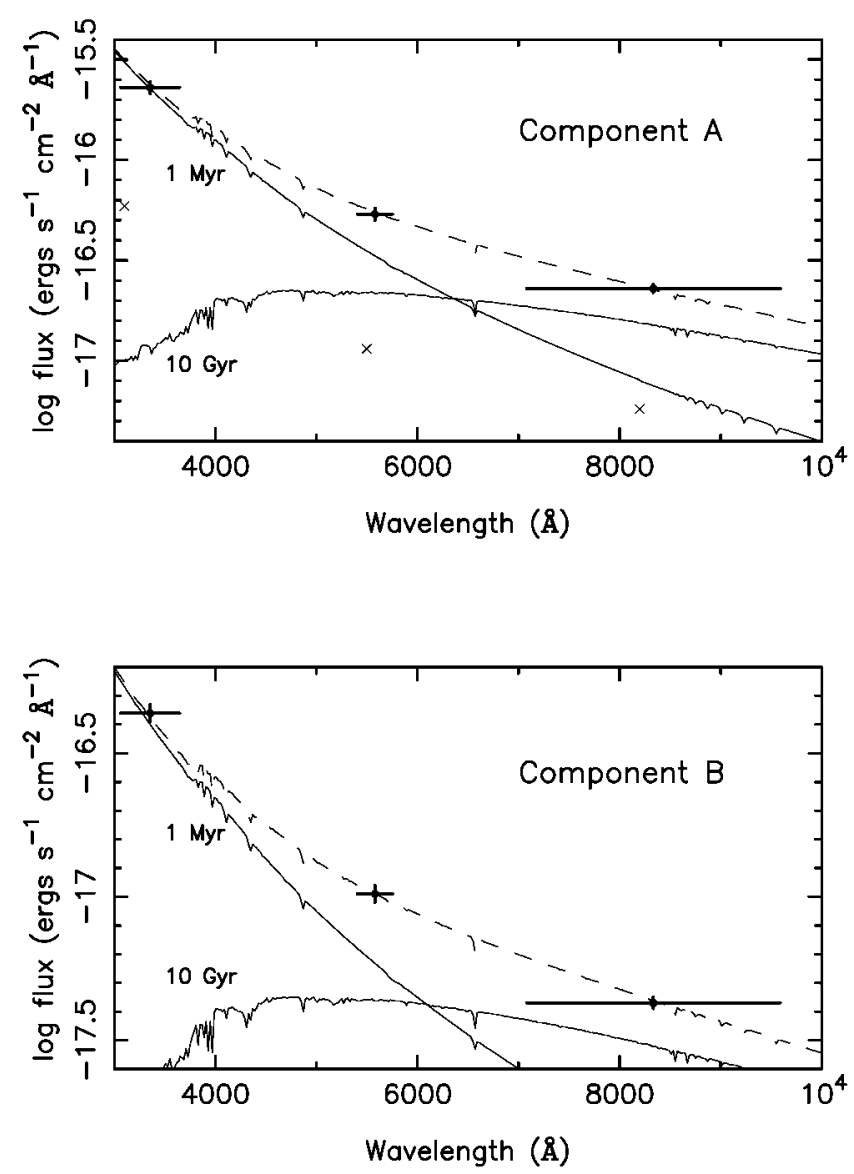

FIG. 2.-Comparison of the F330W, F550M, and F814W fluxes of components A and B with Bruzual \& Charlot (2003) instantaneous burst stellar population synthesis models. The models assume a Chabrier (2003) initial mass function and a metallicity of $Z=0.0004$. The dashed line represents the addition of the $1 \mathrm{Myr}$ model and the $10 \mathrm{Gyr}$ model. The mult crosses shown in the plot for component A represent the estimated combined $\mathrm{H}$ and $\mathrm{He}$ nebular continuum flux $(\S 2.2)$. presented by $\mathrm{P} 03$ at the corresponding wavelength. We attempted to fit these fluxes using Bruzual \& Charlot (2003) population synthesis models. The models chosen represent a simple stellar population (instantaneous burst), a Chabrier (2003) initial mass function, and a metallicity of $Z=0.0004$. This metallicity corresponds to approximately $1 / 48 Z_{\odot}$, which is the closest in the Bruzual \& Charlot (2003) library to that measured for HS $0822+3542$. We find that the fluxes cannot be fitted by a single burst model of any age nor can they be fitted by a model of continuous star formation. They can, however, be fitted to within errors by the addition of a young (a few megayears old) component and an underlying older (several gigayears old) component. Figure 2 shows the result for the addition of the $1 \mathrm{Myr}$ and $10 \mathrm{Gyr}$ models, which fits the measured fluxes well $\left(\chi^{2} \simeq 1\right)$. More precise constraints on the ages of the respective components requires additional optical and near-infrared fluxes of each component, optimally provided by spectra. In particular, there may be a contribution from a population of intermediate-age ( $1 \mathrm{Gyr})$ stars, as has been determined from analyses of the spectra of blue compact dwarf galaxies (Kong et al. 2003; Westera et al. 2004).

The F330W, F550M, and F814W filters exclude the stronger emission lines in the spectrum of the object. However, the nebular continuum emission at these wavelengths can be significant during the first few megayears of a starburst (Leitherer \& Heckman 1995). To address this question, we estimated the flux of the combined $\mathrm{H}$ and $\mathrm{He}$ nebular continuum surrounding the starburst region of component A near the central wavelengths of the filters. Using the emission-line fluxes measured from the Sloan Digital Sky Survey spectrum, we find nebular temperatures and densities of $T \simeq 20,000 \mathrm{~K}$ and $N_{e} \simeq 390 \mathrm{~cm}^{-3}$, respectively. The specific values of the combined $\mathrm{H}$ and $\mathrm{He}$ nebular continuum fluxes that we obtain by assuming $55 \mathrm{O} 7 \mathrm{~V}$ stars as estimated above are plotted with mult crosses in Figure 2. Comparing these values with the fluxes measured in each filter shows that the combined $\mathrm{H}$ and $\mathrm{He}$ nebular continuum emission may contribute significantly $(\sim 10 \%-20 \%)$ to the total flux in each filter but does not dominate it. In particular, it does not seem possible to produce the flux measured in the F814W filter without a significant population of evolved stars. We thus conclude that the emission measured in each filter and seen in Figure 1 is mainly stellar.

There is a large difference between the surface brightnesses of components $\mathrm{A}$ and $\mathrm{B}$ and the surrounding faint plume of stars. We measure a surface brightness of approximately 23 mag $\operatorname{arcsec}^{-1}$ in the F814W filter, where the plume is brightest, after subtracting off the background level measured from a region away from the plume. By contrast, the surface brightness of component $\mathrm{B}$ and the ring of component $\mathrm{A}$ is approximately $20.6 \mathrm{mag} \operatorname{arcsec}^{-1}$. This factor of $\sim 100$ difference in surface brightness further indicates that $\mathrm{A}$ and $\mathrm{B}$ are physically distinct 
objects, as opposed to star clusters within a single galaxy as discussed by $\mathrm{P} 03$.

\section{DISCUSSION}

The small sizes of components A and B are remarkable, lying between globular clusters and objects previously classified as galaxies. While these components are comparable in size to ultracompact dwarf galaxies found in galaxy clusters (see Drinkwater et al. 2003), they differ in that they reside in a void, are actively star-forming, and do not appear to be the remnants or fragments of larger galaxies. HS $0822+3542$ is morphologically similar to the blue compact dwarf galaxy POX 4 , which also consists of a ringlike primary component and a smaller secondary component (Méndez \& Esteban 1999). These authors interpret the ring and starburst of the primary component of POX 4 to be the result of a penetrating collision by the secondary component, which is also appears to be a plausible explanation of HS $0822+3542$ (see, e.g., Athanassoula et al. 1997 and references therein).

The absolute magnitudes and colors of the components correspond to $B$-band luminosities of approximately $5 \times 10^{6} L_{\odot}$ (component A) and $9 \times 10^{5} L_{\odot}$ (component B). Thuan (1987) estimates the ratio of mass to $B$-band luminosity of blue compact dwarf galaxies to be $2-4$, based on dynamical masses. Using these ratios yields masses of (1-2) $\times 10^{7} M_{\odot}$ (component A) and (2-4) $\times 10^{6} M_{\odot}$ (component B). Combining their projected separation of approximately $80 \mathrm{pc}$ with these masses yields a free-fall time for the components of $\sim 2-5 \mathrm{Myr}$. This is short enough for a collision between them to have triggered their starbursts. Interaction between A and B before their recent collision could also have created the surrounding plume of stars through tidal effects, likely with B being tidally stripped by A. In addition, the evidence that the point sources identified around A and B are supergiant stars at the same distance indicates that stars were forming in the plume as recently as $\sim 10^{7} \mathrm{yr}$ ago, which is consistent with the dynamical timescale of the $\mathrm{A} / \mathrm{B}$ system based on the extent of the plume and the above mass estimates.

This interpretation differs from that offered by $\mathrm{P} 03$, who argue that HS $0822+3542$ 's star formation was triggered by interaction with SAO $0822+3545$ between $\sim 0.2$ and $0.3 \mathrm{Gyr}$ ago. Such an interaction may have occurred, and may have contributed to the formation of the stellar plume, but the interaction of A and B seems likely to dominate the recent star formation of the system. It seems likely that components A and B along with the surrounding stars in the plume will eventually coalesce into a single very low mass and quiescent dwarf elliptical galaxy. A similar interpretation was offered by Corbin \& Vacca (2002) for POX 186, which has remarkably similar properties to HS $0822+3542$, including in size, mass, and environment.

The evidence that components A and B contain a population of stars $\sim 10$ Gyr old (Fig. 2) is consistent with the general finding that blue compact dwarf galaxies contain both young and old stars (e.g., Raimann et al. 2000; Kong et al. 2003; Westera et al. 2004). This finding is of particular interest in view of recent evidence that the prototypical extremely metal-poor galaxy I Zw 18 contains no stars older than $\sim 1$ Gyr (Izotov \& Thuan 2004; Östlin \& Mouhcine 2005). This suggests that the similar metal deficiency of HS $0822+3542$ is the result of its low mass and the consequent loss of chemically enriched gas via supernova ejection (see Mac Low \& Ferrara 1999; Martin et al. 2002), as opposed to youth. Extreme metal deficiency among blue compact dwarf galaxies thus may not be a unique indicator of youth. However, given the subgalactic size of components A and B and the likelihood of their ultimate coalescence, it may be fair to describe the HS $0822+3542$ system as being a dwarf galaxy in a stage of assembly from smaller components. The masses we estimate for A and B are close to the minimum mass scale for galaxies forming via atomic cooling within cold dark matter halos (White \& Rees 1978), and the formation of dwarf galaxies in voids was predicted by early cold dark matter models of galaxy formation (Dekel \& Silk 1986). We also note that the H I mass of HS $0822+3542$ is approximately $1.3 \times 10^{7} M_{\odot}$ (J. Chengalur et al. 2005, in preparation, as quoted by P03), which, in comparison with the estimated masses of components $\mathrm{A}$ and $\mathrm{B}$, yields a ratio of gas mass to stellar mass of $\sim 1$. This suggests that the object is still converting much of its gas to stars. Combining the estimate of the object's star formation rate from Kniazev et al. (2000) with our mass estimate yields a high specific or massnormalized star formation rate (SFR; see Kauffmann et al. 2004 and references therein) of $\log \left(\mathrm{SFR} / M_{\odot}\right) \simeq-9.2$ (where SFR is in units of solar mass per year), consistent with this interpretation.

This work was supported by NASA through grant G010180.06-A to Arizona State University from the Space Telescope Science Institute. The Space Telescope Science Institute is operated by the Association of Universities for Research in Astronomy, Inc. under NASA contract NAS 5-26555.

\section{REFERENCES}

Athanassoula, E., Puerari, I., \& Bosma, A. 1997, MNRAS, 286, 284

Bruzual, G., \& Charlot, S. 2003, MNRAS, 344, 1000

Chabrier, G. 2003, PASP, 115, 763

Corbin, M. R., \& Vacca, W. D. 2002, ApJ, 581, 1039

Dekel, A., \& Silk, J. 1986, ApJ, 303, 39

Drinkwater, M. J., Gregg, M. D., Hilker, M., Bekki, K., Couch, W. J., Ferguson, H. C., Jones, J. B., \& Phillipps, S. 2003, Nature, 423, 519

Gil de Paz, A., Madore, B. F., \& Pevunova, O. 2003, ApJS, 147, 29

Izotov, Y. I., \& Thuan, T. X. 2004, ApJ, 616, 768

Kauffmann, G., White, S. D. M., Heckman, T. M., Ménard, B., Brinchmann,

J., Charlot, S., Tremonti, C., \& Brinkmann, J. 2004, MNRAS, 353, 713

Kniazev, A. Y., Grebel, E. K., Hao, L., Strauss, M. A., Brinkmann, J., \& Fukugita, M. 2003, ApJ, 593, L73

Kniazev, A. Y., et al. 2000, A\&A, 357, 101

Kong, X., Charlot, S., Weiss, A., \& Cheng, F. Z. 2003, A\&A, 403, 877

Leitherer, C., \& Heckman, T. M. 1995, ApJS, 96, 9
Mac Low, M.-M., \& Ferrara, A. 1999, ApJ, 513, 142

Martin, C. L., Kobulnicky, H. A., \& Heckman, T. M. 2002, ApJ, 574, 663

Méndez, D. I., \& Esteban, C. 1999, AJ, 118, 2723

Östlin, G., \& Mouhcine, M. 2005, A\&A, 433, 797

Pustilnik, S. A., Kniazev, A. Y., Pramskij, A. G., Ugryumov, A. V., \& Masegosa, J. 2003, A\&A, 409, 917 (P03)

Raimann, D., Bica, E., Storchi-Bergmann, T., Melnick, J., \& Schmitt, H. 2000, MNRAS, 314, 295

Schlegel, D. J., Finkbeiner, D. P., \& Davis, M. 1998, ApJ, 500, 525

Thuan, T. X. 1987, in Nearly Normal Galaxies, ed. S. M. Faber (New York: Springer), 67

Westera, P., Cuisinier, F., Telles, E., \& Kehrig, C. 2004, A\&A, 423, 133

White, S. D. M., \& Rees, M. J. 1978, MNRAS, 183, 341

Whitmore, B. 2003, in Extragalactic Globular Cluster Systems, ed. M. KisslerPatig (Berlin: Springer), 336 